\title{
Cerebral venous malformation with meningioma: A case report
}

\author{
QINGCHUN MU ${ }^{1-3}$, KUN ZHANG $^{3}$, JUSTIN WANG ${ }^{4}$, ARASH SAYARI $^{4}$ and HAIYAN HUANG ${ }^{1}$ \\ ${ }^{1}$ Department of Neurosurgery, The First Hospital of Jilin University, Changchun, Jilin 130021; \\ ${ }^{2}$ Department of Neurosurgery, Hongqi Hospital of Mudanjiang Medical University, Mudanjiang, Heilongjiang 157011; \\ ${ }^{3}$ Cerebrovascular Center, Henan Provincial People's Hospital, Zhengzhou University, Zhengzhou, Henan 450000, P.R. China; \\ ${ }^{4}$ Department of Orthopedics, Keck School of Medicine, University of Southern California, Los Angeles, CA 90089, USA
}

Received March 9, 2015; Accepted October 29, 2015

DOI: $10.3892 /$ etm.2016.3007

\begin{abstract}
A 43-year-old female patient was admitted to The First Hospital of Jilin University (Changchun, China) on 1st October 2011 with a 10-day history of discontinuous, whole-brain headache and a 1-year history of impaired vision and memory deterioration, accompanied by right facial numbness. Clinical signs and radiological features observed using magnetic resonance imaging (MRI) led to the diagnosis of an intracranial meningioma accompanied by a cerebral venous malformation (CVM). The patient underwent neurosurgical resection of the meningioma, but required no further treatment for the CVM. At a 1-year follow-up examination, the patient continued to complain of discontinuous headache. Digital subtraction angiography (DSA) was used to reconfirm the CVM diagnosis; however, no treatment was administered due to the high risks of treatment and only mild symptoms experienced by the patient. The present case demonstrates the efficacy of DSA for detecting the presence and specific nature of CVM, and compares the value of MRI and DSA in the diagnosis of CVM. The majority of CVM patients exhibit no clinical symptoms, and the disease prognosis is typically favorable.
\end{abstract}

\section{Introduction}

Cerebral venous malformations (CVMs) are the most frequently encountered type of cerebral vascular malformation, with an incidence of 0.26 per year worldwide (1). Patients with CVMs may present with serious symptoms, including seizures and subarachnoid and intraparenchymal

Correspondence to: Dr Qingchun Mu, Department of Neurosurgery, Hongqi Hospital of Mudanjiang Medical University, 5 Tongxiang Road, Mudanjiang, Heilongjiang 157011, P.R. China

E-mail: muqcns@gmail.com

Dr Haiyan Huang, Department of Neurosurgery, The First Hospital of Jilin University, 71 Xinmin Street, Changchun, Jilin 130021, P.R. China

E-mail: huanghy@jlu.edu.cn

Key words: cerebral venous malformation, cavernous malformation, magnetic resonance imaging, angiography, morphology, prognosis bleeds; however, $\sim 33 \%$ of patients with CVMs are asymptomatic (2) and thus CVMs are not easily diagnosed, being frequently identified during autopsy (3). CVM lesions are commonly located in the white matter in the brain, such as in the cerebral hemisphere, cerebellum or corpora quadrigemina, and may additionally occur under the pia mater. In the present study, a typical case of CVM is reported. The patient was admitted to The First Hospital of Jilin University (Changchun, China) with a mild and discontinuous headache and a left quadrantanopia. The authors of the present study hypothesized that the impaired vision may have been associated with CVMs, which in turn were caused by a meningioma. If the surface veins of a territory fail to meet the drain, the blood must drain deeply or farther into an unblocked escape channel. Therefore, a number of collecting veins may coalesce deeply to form a draining vein (4); thus suggesting that meningiomas may be considered a contributing factor to the formation of CVMs. It has been shown that obstruction during venous development may result in embryonal medullary veins draining into a few expended draining veins (4). The present study hypothesized that the impaired vision was associated with CVMs, and aimed to raise awareness and to better understand their hemodynamic effects on adjacent areas. The majority of patients with CVMs typically recover well following conservative treatment.

\section{Case report}

A 43-year-old woman with a 3-year history of discontinuous whole-brain headache and a 1-year history of impaired vision and memory deterioration accompanied by right facial numbness was admitted to The First Hospital of Jilin University on 1st October 2011 for treatment following aggravation of the headache for 10 days. Physical examination indicated no neurological deficits. Following admission, the patient underwent non-contrast computed tomography, which showed a round, slightly high-density shadow with well-defined margins $(2.5 \times 3.0 \times 4.1 \mathrm{~cm})$ in the right frontal cortex. Routine enhanced head magnetic resonance imaging (MRI) showed a markedly enhanced lesion adhering to the dura mater, causing mild compression of the right cerebral ventricle (Fig. 1). Several dilated medullary veins in the right occipital lobe converged in a fan shape to form two dilated central veins, with an umbrella- or medusa-like appearance. The diagnosis 
A

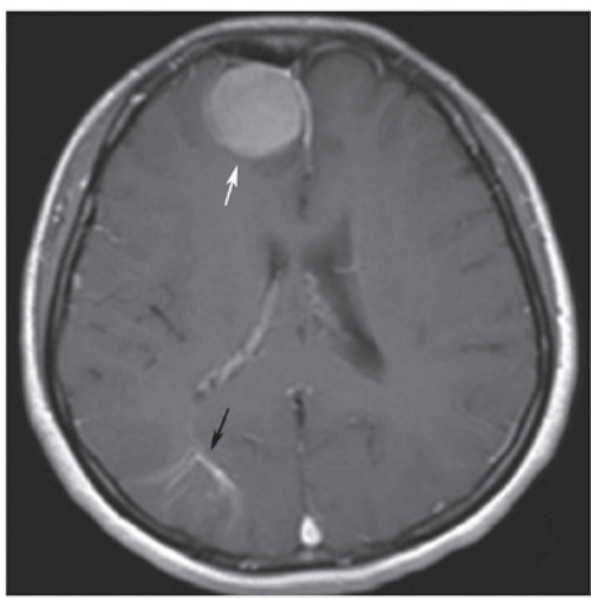

B

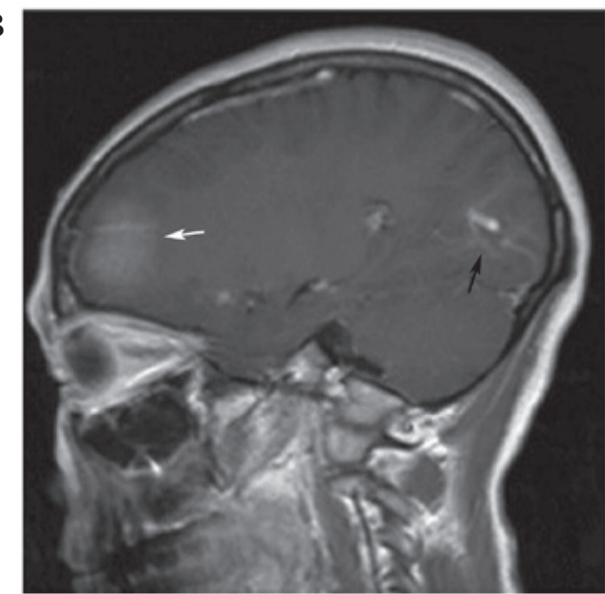

Figure 1. Magnetic resonance T1-weighted (1.5T) images of the brain with intravenous contrast showing cerebral venous malformation coexisting with the frontal meningioma (white arrow). (A) Axial and (B) sagittal planes. Numerous dilated medullary veins drained into the enlarged transcortical collector vein (black arrow).
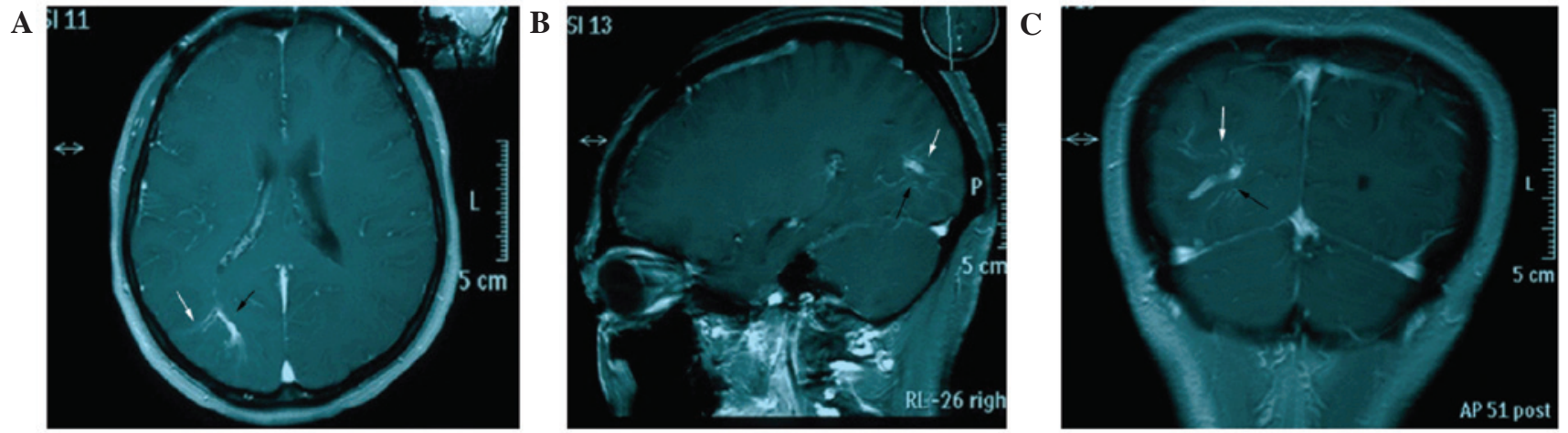

D

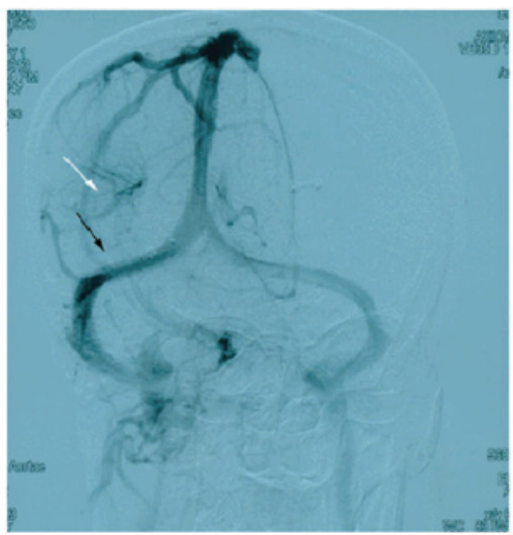

$\mathbf{E}$

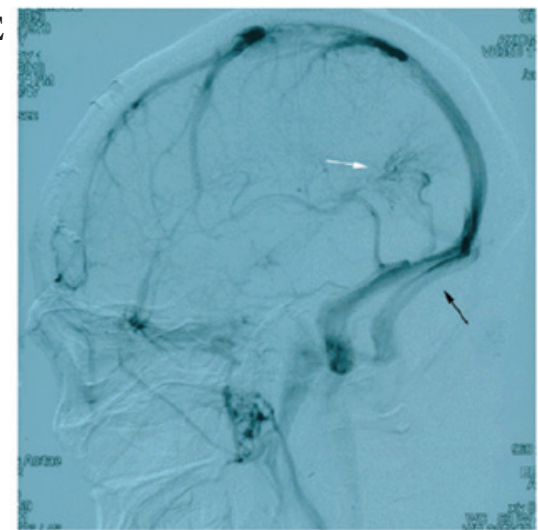

Figure 2. Magnetic resonance (1.5T) T2-weighted images in the (A) axial, (B) sagittal and (C) coronal planes showing markedly dilated veins in the right parietal and occipital lobe (white arrow). Numerous dilated medullary veins drained into the enlarged transcortical collector vein (black arrow). (D and E) Right internal carotid artery injection of the cerebral angiogram showing the caput medusa of the developmental venous anomaly. (D) Frontal and (E) lateral projections of the venous phase show an extensive cerebellar venous malformation with the typical appearance of the caput medusa (white arrow), which is draining into the right transverse sinus (black arrow).

upon admission was intracranial meningioma of the right frontal lobe with concomitant ipsilateral occipital venous malformation. Subsequently, the patient underwent microsurgery for meningioma resection, during which the entire tumor and affected dura mater were resected, and electrocoagulation was repeatedly performed on the basilar dura mater. An MRI demonstrated an ill-defined hyperdensity in the right cerebellar hemisphere. The patient was then discharged from the hospital, with no further treatment required for CVM.
The patient was readmitted to the hospital at 1 year after surgery for follow-up and continued to complain of a mild, discontinuous headache. Enhanced head MRI was evaluated with gadolinium ethoxybenzyl diethylenetriamine pentaacetic acid and digital subtracting angiography (DSA) was performed, which revealed enhancement of the dilated medullary veins and draining veins, presenting in a typical caput medusa-like appearance with a number of small dilated medullary veins converging into a single draining vein in a radial pattern. 
These draining veins drained into the right transverse sinus, which in turn drained into the cavernous sinus through the superficial middle cerebral veins. Furthermore, the DSA showed prolonged vein phase. Fig. 2 shows the drainage direction of the draining veins. No treatment was performed for the CVM due to the high risks of treatment and the paucity of symptoms experienced by the patient. The patient returned to the clinic 8 weeks later and had remained asymptomatic. Written informed consent was obtained from the patient for the publishing of the present study.

\section{Discussion}

CVM is a congenital cerebral vascular disease, which is also known as a brain development venous abnormality (1). It is a type of cerebral vascular malformation histologically composed of venous components, including small dilated medullary veins and one or more draining veins (5). Abnormal dilation of the cerebral veins may be observed, and blood vessel walls contain internal elastic fibers with normal brain tissues between them (6). CVM may occur in any region of the brain, but most commonly originates adjacent to the anterior-inferior cerebellar artery, middle cerebral artery and galenic venous system. CVM is a rare congenital disease, the underlying mechanism of which is not clear $(4,7)$. It is generally considered to be caused by obstruction during venous development, which occurs following the development of the arterial system, resulting in embryonal medullary veins draining into a single thick draining vein (2). Normal island-like areas of brain tissue may exist among these abnormally dilated plexiform veins without feeding arteries or direct arteriovenous shunts (4).

As a CVM develops as venous hypoplasia during the embryonic period, its impact on brain blood circulation is a slow process, allowing for an occult clinical presentation (8). Patients with a CVM may exhibit symptoms including seizure, subarachnoid hemorrhage or intracerebral hemorrhage (9); however, $233 \%$ of CVM patients are asymptomatic (10). A previous study reported that the incidence of intracranial hemorrhage is increasing, primarily due to the coexistence of mixed venous-cavernous hemangiomas and arteriovenous malformations (11). Furthermore, previous autopsy reports indicate that CVMs account for 2.5-2.6\% of cerebral vascular malformations, which is 3-4 times more common than arteriovenous malformations $(8,10,12)$.

CVMs are divided into two types based on the type of draining vein, namely superficial or deep CVM (13), with the present case belonging to the superficial type. Certain researchers indicate that CVM should be considered when MRI reveals a jellyfish head-shaped medullary vein, with a long T1 and long or short T2 signal, or when a large strip-shaped area of no signal appears (12). The use of enhanced MRI scanning to identify these malformed vessels based on their jellyfish or umbrella shape may completely substitute DSA examination $(1,14)$. Under MRI, the T1-weighted image exhibits a flow void signal in patients without ischemic or hemorrhagic complications within the drainage territory of CVMs, while the vessels parallel to the scanning plane exhibit a high signal, combined with a low signal in the T2-weighted image, which is associated with the echo phase reunion phenomenon (12).
Vessels in other directions predominantly exhibit a low signal, the draining veins are quite thick, and the detection rate of MRI is high, which are necessary conditions for CVM diagnosis (15). In enhanced MRI scanning, CVM mainly presents with a markedly high signal and its structure is clear. Enhanced scanning is particularly crucial if the lesion is small, and there is no edema or mass effect around the lesion under MRI (14). A recent publication suggested that three types of 'arterialized developmental venous anomalies (DVAs)' may exist (6), and that DSA is required to adequately characterize arterialized DVAs (14). The standards of DSA diagnosis of CVM are as follows $(14,16)$ : i) Vascular lesion appears in the venous phase, with no feeding artery; ii) numerous small dilated medullary veins; and iii) drainage through a dilated brain-penetrating vein (superficial type) or subependymal vein (deep type). Although MRI is able to diagnose the majority of CVMs, the DSA remains the best imaging module for investigating the hemodynamic behavior of CVMs.

Active surgical treatment is recommended only for CVMs with posterior fossa bleeding (15). For patients with bleeding, craniotomy hematoma evacuation or intraventricular hematoma evacuation may be performed, and patients typically recover well following surgery $(17,18)$. However, the CVM itself is not directly treated as the risk of further bleeding following surgery is high, and resection of the lesion may immediately cause venous infarction of cerebral tissues, leading to edema and congestion of cerebral tissues, or even cerebral necrosis (4).

In conclusion, CVM may be diagnosed using MRI, but DSA remains the best imaging module for investigating the hemodynamic behavior of DVAs. For patients exhibiting bleeding, only craniotomy hematoma evacuation or intraventricular hematoma evacuation is required, while treatment for the CVM may not be necessary in other cases. The majority of patients with CVMs have no clinical symptoms, and prognosis is typically favorable.

\section{References}

1. San Millán Ruíz D, Gailloud P: Cerebral developmental venous anomalies. Childs Nerv Syst 26: 1395-1406, 2010.

2. Jagadeesan BD, Delgado Almandoz JE, Moran CJ and Benzinger TL: Accuracy of susceptibility-weighted imaging for the detection of arteriovenous shunting in vascular malformations of the brain. Stroke 42: 87-92, 2011.

3. Sarwar M and McCormick WF: Intracerebral venous angioma. Case report and review. Arch Neurol 35: 323-325, 1978.

4. Pereira VM, Geibprasert S, Krings T, Aurboonyawat T, Ozanne A, Toulgoat F, Pongpech S and Lasjaunias PL: Pathomechanisms of symptomatic developmental venous anomalies. Stroke 39: 3201-3215, 2008.

5. Vogl TJ, Bergman C, Viliringer A, Einhäupl K, Lissner J and Felix R: Dural sinus thrombosis: Value of venous MR angiography for diagnosis and follow up. AJR Am J Roentgenol 162: 1191-1198, 1994

6. San Millán Ruíz D, Delavelle J, Yilmaz H, Gailloud P, Piovan E, Bertramello A, Pizzini F and Rüfenacht DA: Parenchymal abnormalities associated with developmental venous anomalies. Neuroradiology 49: 987-995, 2007.

7. Bisdorff A, Mulliken JB, Carrico J, Robertson RL and Burrows PE: Intracranialvascular anomalies in patients with periorbital lymphatic and lymphaticovenous malformations. AJNR Am J Neuroradiol 28: 335-341, 2007.

8. Leblanc GG, Golanov E, Awad IA and Young WL; Biology of Vascular Malformations of the Brain NINDS Workshop Collaborators: Biology of vascular malformations of the brain. Stroke 40: e694-e702, 2009. 
9. Ruíz DS, Yilmaz H and Gailloud P: Cerebral developmental venous anomalies: Current concepts. Ann Neurol 66: 271-283, 2009.

10. Vattoth S, Purkayastha S, Jayadevan ER and Gupta AK: Bilateral cerebral venous angioma associated with varices: A case report and review of the literature. AJNR Am J Neuroradiol 26: 2320-2322, 2005.

11. Numaguchi Y, Nadell JM, Mizushima A and Wilensky MA: Cerebral venous angioma and a varix: A rare combination. Comput Radiol 10: 319-323, 1986.

12. Saeed O, Khan AA, Herial NA and Qureshi AI: Exercise Induced Transient Neurological Deficit in a Patient with Cerebellar Developmental Venous Anomaly. J Vasc Interv Neurol 8:17-20, 2015.

13. Dross P, Raji MR and Dastur KJ: Cerebral varix associated with a venous angioma. AJNR Am J Neuroradiol 8: 373-374, 1987.

14. Ostertun B and Solymosi L: Magnetic resonance angiography of cerebral developmental venous anomalies: Its role in differential diagnosis. Neuroradiol 35: 97-104, 1993.
15. Santucci GM, Leach JL, Ying J, Leach SD and Tomsick TA: Brain parenchymal signal abnormalities associated with developmental venous anomalies: Detailed MR imaging assessment. AJNR Am J Neuroradiol 29: 1317-1323, 2008.

16. Guclu B, Ozturk AK, Pricola KL, Seker A, Ozek M and Gunel M: Cerebral venous malformations have distinct genetic origin from cerebral cavernous malformations. Stroke 36: 2479-2480, 2005.

17. Wilums G, Demaerel P, Marchal G, Baert AL and Plets C: Gadolinium-enhanced MRI of cerebral venous angiomas with emphasis on their drainage. J Comput Assist Tomogr 15: 199-206, 1991.

18. Cakirer S: De novo formation of a cavernous malformation of the brain in the presence of a developmental venous anomaly. Clin Radiol 58: 251-256, 2003. 